\title{
Matriz de competências para o ensino da homeopatia na graduação médica
}

\author{
Competency matrix for undergraduate medical education in homeopathy
}

\author{
Francisco José de Freitas ${ }^{1}$ (1) $\mid$ fdefreitas@gmail.com \\ Rodrigo da Fontoura de Albuquerque Mello' 1 (i) rodrigofmello2000@yahoo.com.br \\ Maria Tereza Serrano Barbosa' (D) tserranobarbosa@gmail.com
}

\begin{abstract}
RESUMO
Introdução: Currículos médicos de graduação são extremamente carregados em seu conteúdo, tornando-se primordial a necessidade de otimizar competências essenciais. As Diretrizes Curriculares Nacionais (DCN) do Curso de Graduação em Medicina preconizam uma valorização do tratamento do doente que possui muitas interfaces com a homeopatia. Apesar de ser uma especialidade médica no Brasil reconhecida desde 1980, a homeopatia ainda é pouco presente na graduação médica.
\end{abstract}

Objetivo: Este estudo teve como objetivo desenvolver uma matriz de competências essenciais composta de conhecimentos e habilidades desejáveis para o ensino da homeopatia na graduação médica.

Métodos:Trata-se de um estudo quanti-qualitativo realizado por meio da técnica Delphi normativa, em duas rodadas, com os docentes especialistas em homeopatia do país. Na primeira rodada, aplicou-se um questionário anônimo on-line para identificar as competências (conhecimentos e habilidades) necessárias em homeopatia para os discentes de graduação em Medicina. Após a análise de conteúdo, os temas foram agrupados nessas duas categorias que retornaram para apuração na segunda rodada, em que os especialistas assinalaram o grau de concordância (escala de Likert de quatro pontos: de não relevante a muito relevante). Para a definição de consenso, adotaram-se dois critérios: índice de De Loe e uma nota de relevância adotada pelos autores com vistas a discriminar mais os graus de consenso.

Resultados: Dos 14 temas/subtemas relacionados como competências/conhecimentos, consideraram-se 11 como de alto consenso, dos quais cinco foram avaliados como primordiais, pois obtiveram nota de relevância acima de 9,0 (três relacionados ao grande tema clínica homeopática; um, à teoria e técnica homeopáticas; e outro, à pesquisa homeopática). Em relação às competências/habilidades, oito das 11 foram consideradas de alto consenso, e somente duas alcançaram nota acima de 9,0, ambas relacionadas à clínica homeopática.

Conclusão: O ensino da homeopatia pode contribuir para a mudança paradigmática da medicina no sentido de valorizar o doente e promover a saúde, permitindo uma atuação médica mais humanizada e centrada no doente, o que justificaria a adoção de seu ensino, inclusive obrigatório, nas faculdades de Medicina. A elaboração de uma matriz de competências do que deve ser ensinado de homeopatia aos discentes da graduação médica vem ao encontro das DCN e instrumentaliza a reflexão na elaboração de uma futura ementa.

Palavras-chave: Homeopatia; Matriz de Competências; Educação de Graduação em Medicina; Educação Baseada em Competências; Currículo.

\begin{abstract}
Introduction: Undergraduate medical curricula are overloaded with content, making the need to optimize essential skills and knowledge paramount. The National Curricular Guidelines for the medical course (DCNs) put focus on patient treatment, which involves several interfaces with homeopathy. However, despite it being recognized as a medical specialty in Brazil since 1980, homeopathy is rarely present in undergraduate medical education.
\end{abstract}

Objective: To develop a collection of essential skills and knowledge for teaching homeopathy as part of the undergraduate medical curriculum.

Methods: Quanti-qualitative study performed using the standard Delphi technique in two rounds with specialized homeopathy teachers from Brazil. An anonymous online questionnaire was conducted to identify the learning competences needed in homeopathy for undergraduate medical students. Following content analysis, the themes and sub-themes were grouped into the two main categories of knowledge and skills, which were returned to the specialists who rated their importance for each item using the "Four-Point Likert Scale" from not at all important to very important. For the purpose of generating a general consensus, two criteria were adopted: the "De Loe index" and a importance score.

Results: There was a high degree of consensus regarding eleven of the fourteen themes/sub-themes, five of which were considered essential, with an importance score above 9.0 (three related to the general theme, Homeopathic Clinic, one to Homeopathic Theory and Method and another to Homeopathic Research). There was also a high level of consensus regarding eight of the eleven skills, but only two gained a score of above 9.0, both related to Homeopathic Clinic.

Conclusion: The teaching of Homeopathy can contribute to a paradigmatic change in medicine, particularly in the sense of prioritizing the patient and promoting health, as well as allowing for a more humanized and patient-centered medical engagement. These factors would justify the adoption of its teaching, so much so it could be made mandatory across all medical study. The establishment of a "competency matrix" for homeopathy study, which ought to be taught to students of the medical field, meets the DCNs and would be instrumental in future syllabuses.

Keywords: Homeopathy; Competency Matrix; Undergraduate Education in Medicine; Competency Based Education; Curriculum.

1 Universidade Federal do Estado do Rio de Janeiro, Rio de Janeiro, Rio de Janeiro, Brasil.

Editora-chefe: Rosiane Viana Zuza Diniz ～～Editor associado: Pedro Tadao Hamamoto Filho

Recebido em 27/09/20; Aceito em 24/11/20. Avaliado pelo processo de double blind review. 


\section{INTRODUÇÃO}

As atuais Diretrizes Curriculares Nacionais (DCN) do Curso de Graduação em Medicina, reformuladas pela Resolução do Conselho Nacional de Educação e da Câmara de Educação Superior (CNE/CES) n 3, de 20 de junho de 2014', reforçam a integração entre a educação superior e a saúde, em consonância com os princípios, os objetivos e as diretrizes do Sistema Único de Saúde (SUS) ${ }^{2}$ e a necessidade de um perfil de egresso com formação geral, humanista, crítica, reflexiva e ética com destaque para a integralidade e humanização do cuidado, a comunicação por meio de linguagem verbal e não verbal, e o cuidado centrado na pessoa, na família e na comunidade. Essas diretrizes recomendam que as instituições de ensino superior (IES), a fim de contemplarem as necessidades do SUS, organizem, desenvolvam e avaliem a formação médica baseada no ensino por competências, compreendidas como a capacidade de mobilizar e integrar conhecimentos, habilidades e atitudes para resolver problemas ${ }^{3}$.

Segundo Marinho-Araujo e Rabello ${ }^{4}$, características subjetivas tais como capacidades cognitivas, afetos, desejos, saberes, conceitos, posturas e atitudes também fariam parte do termo competências.

Portanto, determinar competências para a construção de uma matriz não é tarefa das mais simples. $O$ ensino por competências em um curso de Medicina implicaria desenvolver no estudante a capacidade de mobilizar conhecimentos, habilidades e atitudes para lidar com situações, problemas e dilemas da vida real. Geralmente, as matrizes curriculares não conseguem apresentar claramente seus conteúdos prioritários, pois a maioria dos professores, especialistas e pesquisadores tem dificuldades, no concerne aos discentes, em aproximar a parte acadêmica da assistencial com uma abordagem predominantemente especializada em detrimento daquela necessária para a formação de cunho generalista, o que gera um aumento do conteúdo da disciplina com temas não essenciais. Para dirimir o complexo trabalho de ensino-aprendizagem, a busca por um ideal de ensino aponta para a necessidade de construção de uma matriz de competências médicas que exerça a função, de forma clara, dos consensos coletivos acerca dos conteúdos primordiais no processo de formação profissional, daquilo que é imprescindível. Gontijo et al. ${ }^{5}$ esclarecem esse desejo ao sinalizarem que uma matriz de competências é de responsabilidade coletiva, e só dessa forma haverá uma expressa legitimação social de estudantes que passarão a ser reconhecidos com capacidade de atuar na carreira médica.

Os currículos médicos de graduação são extremamente carregados em seu conteúdo, e a prática médica predominante privilegia o tratamento da doença em detrimento do doente, tornando-se primordial a necessidade de se otimizarem as competências essenciais a serem alcançadas pelos discentes de qualquer disciplina.

Todavia, o fato de a prática médica predominante privilegiar o tratamento da doença em detrimento do paciente dificulta a implementação, no ensino de graduação, de paradigmas relacionados às disciplinas e práticas centradas no doente, tais como a medicina tradicional chinesa/acupuntura, medicina antroposófica e a disciplina objeto deste artigo, a homeopatia ${ }^{6-11}$.

O ensino e a prática da homeopatia possuem muitas interfaces com aquelas preconizadas pelas DCN, as quais possibilitam ao egresso médico uma melhor atuação no que tange à decisão da conduta terapêutica mais adequada nos campos da reabilitação, recuperação, prevenção e/ou promoção voltadas à saúde do doente, levando em conta cada caso individualmente, de modo a estabelecer não somente uma relação médico-paciente mais completa e humanizada, inclusive com toda a família do paciente, mas também com o intuito de valorizar a interação com profissionais de outras especialidades ${ }^{9,12-14}$.

Existe um consenso entre os docentes de quais competências esses discentes deveriam alcançar? Todas essas questões tornam-se ainda mais agudas nas disciplinas humanísticas, como no caso específico da homeopatia, que ainda não se encontram suficientemente valorizadas e presentes no contexto da educação médica ${ }^{10,14,15}$.

Para se obter consenso, parte-se da premissa de que uma avaliação precisa e confiável pode ser mais bem alcançada quando se consulta um painel de especialistas e se aceita o consenso do grupo, pela sua suposta capacidade de extrair o conhecimento coletivo da profissão, que, muitas vezes, é implícito e difícil de ser verbalizado e formalizado ${ }^{16}$.

Apesar de alguns trabalhos demonstrarem um bom grau de satisfação e aceitação do ensino da homeopatia na graduação no Brasil e no exterior ${ }^{7-9,13}$, nossa pesquisa não encontrou trabalhos que apontem efetivamente o conteúdo a ser ministrado nessa disciplina.

Diante da realidade do ensino da homeopatia na graduação médica - quando existe, é, na sua grande maioria, sob a forma de disciplina optativa e quase exclusivamente teórica, e com pequena carga horária -, e também com o propósito de tentar equacionar a questão do ensino médico adequado ao que é recomendado pelas DCN, este trabalho objetiva alcançar, por meio da técnica Delphi, um consenso dos especialistas, professores de homeopatia da graduação e pós-graduação médicas no Brasil a respeito do que deveria ser ensinado aos discentes da graduação de Medicina, a fim de desenvolver uma matriz de competências essenciais, composta de conhecimentos e habilidades desejáveis no que se relaciona à ciência homeopática. 


\section{MÉTODOS}

A técnica Delphi é um estudo exploratório quantiqualitativo que tem como objetivo obter consenso sobre determinadas questões, principalmente quando os dados sobre o assunto tratado são escassos, com a formação de uma opinião coletiva qualificada ${ }^{5,17}$.

É uma ferramenta de pesquisa que assegura o anonimato dos participantes e, dessa forma, obtém opiniões mais isentas dos especialistas. Há três tipos de estudo Delphi: convencional, normativo e político. O Delphi convencional concentra-se em uma opinião de grupo sobre determinado assunto, muitas vezes associada a previsões de algum tipo. $\mathrm{O}$ Delphi normativo busca identificar e estabelecer prioridades a fim de estruturar e projetar um determinado assunto. $\mathrm{O}$ Delphi político procura gerar pontos de vista opostos sobre um determinado assunto, normalmente relacionado às políticas e à implementação destas - nesse caso, enfatiza-se a identificação de opiniões divergentes e não o consenso ${ }^{18}$.

Neste estudo, utilizou-se a técnica Delphi normativa não somente com base na justificativa já apresentada, mas também porque a intenção era fazer que um grupo de especialistas desse a sua contribuição para a definição das competências essenciais a seremalcançadas pelos estudantes dehomeopatia. Nessemétodo, sobressaem as vantagens de que as informações e o número de fatores considerados estejam disponíveis e contrastados com aqueles dos outros especialistas - "várias cabeças pensam melhor do que uma"19. De posse dessas informações, é feito um trabalho de representação estatística cujo resultado será distribuído para dar um feedback ao grupo, gerando uma reavaliação do conteúdo, com rodadas subsequentes até a obtenção de um consenso o mais uniforme possível.

\section{População-alvo}

O público-alvo foi constituído por docentes de homeopatia, atuantes na graduação e pós-graduação nas faculdades médicas públicas e privadas do Brasil, por meio de consulta direta a elas e também a partir do Conselho de Entidades Formadoras (CEF) da Associação Médica Homeopática Brasileira (AMHB). Frise-se que a AMHB é uma instituição filiada à Associação Médica Brasileira (AMB) e congrega as entidades médicas homeopáticas em todo território nacional. O CEF possui professores de pelo menos 14 estados: Alagoas, Bahia, Ceará, Distrito Federal, Espírito Santo, Mato Grosso do Sul, Minas Gerais, Paraná, Pernambuco, Rio de Janeiro, Rio Grande do Norte, Rio Grande do Sul, Santa Catarina e São Paulo.

\section{Critérios de inclusão e exclusão}

$\mathrm{Na}$ primeira rodada, incluíram-se na pesquisa todas as respostas dos docentes que preencheram pelo menos
$80 \%$ das perguntas do questionário sociodemográfico e que contribuíram com pelo menos cinco sugestões de competências/conhecimentos e cinco competências/ habilidades. Na segunda rodada, adotou-se o mesmo critério para o questionário sociodemográfico. Os respondentes que não cumpriram esses critérios foram excluídos.

\section{Instrumento e técnica de coleta de dados}

$\mathrm{Na}$ primeira rodada, aplicou-se um questionário exploratório anônimo on-line, utilizando a ferramenta Formulários Google a fim de: 1. identificar as competências, especificamente os conhecimentos e as habilidades, necessárias em homeopatia a serem alcançadas pelos discentes de graduação em Medicina; 2. obter informações do perfil dos especialistas para caracterizar a amostra, como idade, formação acadêmica, experiência docente na graduação e/ou pósgraduação e estado da Federação em que atua, que também foi aplicado na segunda rodada. Após a análise de conteúdo, criam-se grandes temas/domínios de competências, agrupados nestas duas categorias - conhecimento e habilidades.

Para a segunda rodada, a partir da agregação de conhecimentos e habilidades semelhantes, foi elaborado um novo questionário exploratório igualmente anônimo e on-line por meio da ferramenta Formulários Google, para apuração dos temas enviados por todos, oriundos da primeira rodada. Os especialistas assinalaram, então, o grau de concordância em cada questão, a partir de uma escala de Likert de quatro pontos: 1 - não relevante, 2 - pouco relevante, 3 - relevante e 4 - muito relevante. A escala de Likert é uma das mais utilizadas em estudos da área da educação, inclusive médica e na enfermagem, para classificar ou ordenar iten $\mathrm{s}^{20-22}$.

\section{Análise estatística e tratamento dos dados}

Na primeira rodada, submeteram-se os dados à análise de conteúdo, para que fosse possível criar, a partir da identificação de semelhanças, temas e subtemas de competências agrupados nas categorias conhecimentos e habilidades.

Na segunda rodada, para a definição de consenso, adotaram-se dois critérios:

- O primeiro baseou-se no índice de De Loe (de nenhum a alto consenso), conforme descrito no Quadro 1, que é um critério que classifica consenso em quatro categorias (alto, médio, baixo ou nenhum) $)^{20,23}$.

- O segundo critério foi a nota de relevância adotada pelos autores com vistas a discriminar mais os graus de consenso, caracterizados pela concentração das respostas nas categorias muito relevante e relevante. 
Quadro 1.Critério de consenso

\begin{tabular}{|c|c|c|}
\hline \multirow{4}{*}{ De Loe } & $\begin{array}{c}70 \% \text { de respostas em uma } \\
\text { categoria ou } 80 \% \text { em duas } \\
\text { categorias contíguas }\end{array}$ & Alto consenso \\
\hline & $\begin{array}{c}60 \% \text { de respostas em uma } \\
\text { categoria ou } 70 \% \text { em duas } \\
\text { categorias contíguas }\end{array}$ & Médio consenso \\
\hline & $\begin{array}{c}50 \% \text { de respostas em uma } \\
\text { categoria ou } 60 \% \text { em duas } \\
\text { categorias contíguas }\end{array}$ & Baixo consenso \\
\hline & $\begin{array}{c}\text { Menos de } 60 \% \text { em duas } \\
\text { categorias contíguas }\end{array}$ & Nenhum consenso \\
\hline
\end{tabular}

Fonte: Elaborado pelos autores baseado em Shimizu e Fragelli20.

Essa nota de relevância foi calculada a partir de uma média ponderada das frequências de relevâncias com pesos crescentes de um a quatro relacionados aos conceitos de não relevante até muito relevante. Seu objetivo foi atribuir uma nota de 0 a 10,0 para diferenciar as competências que tinham graus de consensos semelhantes.

\section{Aspectos éticos}

O projeto foi aprovado pelo Comitê de Ética em Pesquisa do Hospital Universitário Gaffrée e Guinle (HUGG) da Universidade Federal do Estado do Rio de Janeiro (Unirio) pelo Parecer $n^{\circ}$ 3.358.215. Todos os participantes assinaram o Termo de Consentimento Livre e Esclarecido .

Em ambas as etapas, enviou-se por e-mail uma breve explicação sobre a pesquisa, por meio de uma carta-convite que esclarecia os objetivos e as rodadas do estudo. O participante poderia se recusar a responder a uma ou mais perguntas caso não se sentisse confortável. Foi igualmente esclarecido que os dados coletados poderiam ter seus resultados divulgados em eventos, revistas e/ou trabalhos científicos.

\section{RESULTADOS}

Enviaram-se os questionários a 76 docentes tanto na primeira rodada quanto na segunda, com cerca de $35 \%$ respondentes, 26 docentes na primeira e 27 na segunda.

Como as respostas aos questionários eram anônimas, comparou-se o perfil sociodemográfico dos participantes nas duas rodadas. Não houve diferenças estatisticamente significativas entre elas, e a idade média dos especialistas foi de 54 anos. Entretanto, destaca-se que, na média das duas rodadas, 33\% dos docentes especialistas em homeopatia eram doutores, e 29\%, mestres. Em relação à experiência docente, apenas 35\% tinham menos de dez anos, e a segunda rodada, que incluiu uma questão sobre a atuação docente, mostrou que $77 \%$ atuavam na graduação médica, dos quais metade tinha mais de 20 anos de experiência. Quanto à distribuição geográfica, $70 \%$ dos especialistas identificaram seu estado da Federação: $68,5 \%$ da Região Sudeste e os demais igualmente distribuídos nas Regiões Sul, Centro-Oeste e Nordeste.

$\mathrm{Na}$ primeira rodada, solicitou-se que os participantes listassem pelo menos cinco competências/conhecimentos e cinco competências/habilidades. Essas respostas foram analisadas por semelhanças e agrupadas em um total de 25 temas. Do total obtido, 14 temas relacionados às competências/ conhecimentos e 11 às competências/habilidades retornaram para a segunda rodada e foram classificados quanto ao grau de relevância, conforme a escala de Likert. Segundo a classificação de De Loe, houve um alto grau de consenso tanto nas competências/conhecimentos quanto nas competências/ habilidades, enquanto a nota de relevância atribuída pelos autores permitiu discriminar os graus de relevância.

No Quadro 2, encontram-se classificadas, em ordem decrescente de consenso e de notas de relevância, as competências relacionadas aos conhecimentos, atribuídas pelos especialistas na segunda rodada, aos temas oriundos da primeira rodada.

Onze das 14 competências/conhecimentos foram de alto consenso, sendo cinco consideradas como primordiais, pois obtiveram nota de relevância acima de 9,0. Destas, destacaramse três relacionadas ao grande tema clínica homeopática (competências 1, 4 e 5); uma, à teoria e técnica homeopáticas (2); e outra, à pesquisa homeopática (3). Nas seis restantes, com notas entre 8,0 e 9,0, quatro são relacionadas ao grande tema teoria e técnica homeopáticas (competências 6, 7, 8 e 10); uma, à matéria médica homeopática (9); e outra, à homeopatia e saúde pública (11).

Em relação às competências/habilidades, oito das 11 foram consideradas de alto consenso, conforme mostra o Quadro 3.

Das oito competências relacionadas às habilidades classificadas como consenso alto, somente duas alcançaram nota acima de 9,0, consideradas pelos respondentes como primordiais, ambas relacionadas ao grande tema clínica homeopática (competências 15 e 16).

Além dessas, cinco habilidades obtiveram altos consensos, porém foram consideradas não tão primordiais, pois obtiveram notas de relevância abaixo de 9,0 e acima de 8,0. Destaca-se a classificada em primeiro lugar desse grupo que é o tema relacionado à pesquisa em homeopatia (competência 17), enquanto as outras quatro $(18,19,20$ e 21$)$ pertencem ao grande tema clínica homeopática. 
Quadro 2. Análise dos consensos temáticos sobre competências/conhecimentos conforme os índices de De Loe e as notas de relevância da segunda rodada

\section{Competências/conhecimentos}

\begin{tabular}{ccc}
$\begin{array}{c}\text { frequência em } \\
\text { cada categoria } \\
\text { de relevância }\end{array}$ & $\begin{array}{c}\text { Consenso } \\
\text { De Loe }\end{array}$ & $\begin{array}{c}\text { Nota de } \\
\text { relevância }\end{array}$ \\
\hline $25 / 2 / 0 / 0$ & Alto & 9,8 \\
\hline $24 / 3 / 0 / 0$ & Alto & 9,7 \\
\hline $20 / 7 / 0 / 0$ & Alto & 9,4 \\
\hline $21 / 3 / 2 / 1$ & Alto & 9,1 \\
\hline $18 / 8 / 1 / 0$ & Alto & 9,1 \\
\hline $12 / 13 / 2 / 0$ & Alto & 8,4 \\
\hline $12 / 13 / 1 / 1$ & Alto & 8,3 \\
\hline $11 / 13 / 2 / 1$ & Alto & 8,2 \\
\hline $11 / 12 / 4 / 0$ & Alto & 8,2 \\
\hline $9 / 17 / 0 / 1$ & Alto & 8,2 \\
\hline $11 / 11 / 5 / 0$ & Alto & 8,1 \\
\hline $11 / 9 / 6 / 1$ & Médio & 7,8 \\
\hline $6 / 13 / 3 / 4^{*}$ & Médio & 7,0 \\
\hline $6 / 11 / 7 / 3$ & Baixo & 6,9 \\
\hline
\end{tabular}

1. Entender a semiologia homeopática que individualiza o paciente levando em conta a totalidade sintomática.

2. Identificar e reconhecer os princípios fundamentais da homeopatia.

3. Conhecer os avanços e as evidências científicas em homeopatia.

4. Conhecer e entender a anamnese homeopática e os diagnósticos em homeopatia.

5. Conhecer os conceitos de totalidade sintomática.

6. Identificar os princípios básicos da farmacotécnica homeopática e a origem dos medicamentos homeopáticos.

7. Noções sobre vitalismo e conceito homeopático de saúde e doença.

8. Conhecer o Organon da arte de curar, de Samuel Hahnemann, os princípios da filosofia homeopática e da racionalidade médica homeopática.

9. Conhecer a matéria médica homeopática de alguns medicamentos.

Baixo

10. Correlacionar a história da medicina e da homeopatia, a evolução do pensamento médico de Hipócrates a Hahnemann e a contextualização histórica, social, política, científica e acadêmica da homeopatia.

11. Conhecer a importância da homeopatia no SUS, os princípios da integralidade e da Política Nacional de Práticas Integrativas e Complementares - PNPIC.

12. Conhecer o conceito homeopático de doenças agudas e crônicas (miasmas/diáteses principais - psora, sicose e sifilinismo).

13. Conhecer a aplicação prática das leis de Hering.

14. Conhecer o repertório homeopático de sintomas.

* Um dos participantes não respondeu a essa questão.

Fonte: Elaborado pelos autores.

Quadro 3. Análise dos consensos temáticos competências/habilidades conforme os índices de De Loe e as notas de relevância da segunda rodada

\section{Competências/habilidades}

15. Entender o paciente na sua totalidade sintomática e o processo doença/doente/ saúde como representação da sua singularidade individual sob visão homeopática.

16. Saber indicar o tratamento homeopático e encaminhar o paciente ao médico homeopata e a outros especialistas.

17. Saber ler e analisar criticamente artigos científicos homeopáticos.

18. Realizar a anamnese homeopática desenvolvendo abordagem mais humanística e empática, ouvindo o paciente sem preconceitos, fortalecendo a relação médico-paciente.

19. Saber receber o paciente tratado com a homeopatia e identificar em quais sintomas a homeopatia está intervindo.

20. Saber orientar o tratamento homeopático e a prescrição homeopática.

21. Realizar a anamnese homeopática identificando, valorizando, hierarquizando e diferenciando os sinais e sintomas pertencentes à(s) doença(s) assim como aos pacientes (totalidade sintomática), com as suas peculiaridades.

22. Perceber as evoluções clínicas dos pacientes e tomar medidas preventivas de acordo com os diagnósticos em homeopatia (hipóteses diagnósticas).

Frequência em
cada categoria de
relevância

$19 / 7 / 1 / 0$

\section{Consenso \\ De Loe}

Alto

$19 / 7 / 1 / 0$

19/7/1/0

$16 / 9 / 1 / 0^{*}$

16/9/1/1

$13 / 12 / 2 / 0$

$14 / 8 / 4 / 1$

$13 / 9 / 4 / 1$

$9 / 12 / 5 / 0^{*}$

$10 / 10 / 6 / 1$

$6 / 13 / 7 / 1$

$7 / 10 / 6 / 4$

25 . Saber identificar os sinais e sintomas do
repertório a fim de realizar a repertorização.

Fonte: Elaborado pelos autores.
Nota de relevância

9,2

9,2

8,9

8.7
Médio

7,2 


\section{DISCUSSÃO}

A técnica Delphi, que tem sido utilizada na educação, principalmente nos cursos de Medicina e Enfermagem, não demanda que os painéis de especialistas sejam constituídos por um número grande de participantes. Na literatura, sugere-se dez participantes como o número mínimo ótimo, e alguns autores consideram como representativo um grupo homogêneo bem escolhido e de qualidade que não exceda 30 participantes ${ }^{17,22,24,25}$. Portanto, o número de 26 e 27 docentes especialistas em homeopatia que responderam, respectivamente, à primeira e segunda rodadas, adequou-se à proposta deste trabalho.

Em relação ao perfil desses docentes especialistas, a concentração de $50 \%$ na Região Sudeste vai ao encontro das características identificadas na demografia médica brasileira ${ }^{26}$. Surpreendeu o fato de que $77 \%$ do total dos respondentes atuam na graduação, considerando-se que a homeopatia ainda é uma disciplina alijada dos currículos médicos.

Cabe-se destacar que, apesar de poucas faculdades de Medicina do Brasil possuírem o ensino da homeopatia na sua grade curricular de graduação ${ }^{10,27}$, este pode encontrar-se inserido em uma disciplina afim como Medicina Integrativa, Racionalidades Médicas, Medicina Complementar e Alternativa, Saúde Coletiva, Práticas Integrativas e Complementares (PIC), Saúde e Sociedade ou outras, de caráter optativo, eletivo ou até mesmo obrigatório, como acontece em alguns países da Europa $7,8,27,28$.

A proposta de uma matriz que identifique e defina as competências necessárias para o ensino da homeopatia é uma etapa crítica no processo de construção ${ }^{29}$ do conjunto de valores, saberes e comportamentos que reforcem a boa prática médica com a contribuição dos preceitos homeopáticos.

No processo de construção dessa matriz, por causa do alto grau de consenso obtido na segunda rodada, a partir das competências conhecimentos e habilidades ao final da primeira, adotou-se uma nota de relevância para discriminar as diferenças. Tal estratégia permitiu demonstrar as competências de maior relevância, mesmo entre aquelas apontadas como de consenso alto $^{23}$ (De Loe).

Com isso foi possível identificar as cinco competências/ conhecimentos e as duas competências/habilidades consideradas como imprescindíveis (consenso alto e nota de relevância acima de 9,0), para compor uma matriz curricular, ou seja, altamente recomendáveis que constem no conteúdo a ser ensinado.

Em relação aos subtemas pertencentes ao grande tema clínica homeopática, evidenciados nas competências conhecimentos e habilidades pelos docentes especialistas, verificou-se a importância da contribuição da prática homeopática na abordagem individualizada, humanizada, integral e empática, entre outras, dentro da realidade ambiental, psicossocial, e também na abordagem do doente e na relação médico-paciente ${ }^{1,7-9,12,13,27,28,30}$.

Tal achado demonstra a necessidade, pela visão dos docentes especialistas consultados, que da mesma forma que o egresso médico precisa saber indicar e encaminhar um paciente para uma especialidade clínica especializada também necessita ser igualmente competente para indicar e encaminhar para o tratamento homeopático. São temas intimamente relacionadas à prática, que demanda um serviço assistencial de homeopatia nas IES que venham a oferecer o seu ensino e um espaço logístico para que os discentes possam vivenciar a clínica homeopática. Fica implícito na recomendação da matriz que, além do componente teórico, deve haver também carga horária destinada à prática.

O destaque conferido pelos especialistas ao tema pesquisa em homeopatia, tanto nas competências conhecimentos quanto nas habilidades, sinalizou a importância que atribuem não somente à necessidade de o discente conhecer as evidências científicas, mas também ao fato de ele saber ler criticamente os artigos. Isso traduz uma demanda implícita dos graduandos em Medicina sobre as comprovações de ação da homeopatia e vai ao encontro das DCN em diversas seções, principalmente no que compete à tomada de decisões baseadas em evidências científicas ${ }^{1,7}$.

Cabe ressaltar que, apesar da consonância da homeopatia com as DCN e com os princípios do SUS, além do fato de esse método terapêutico estar contido na PNPIC, evidenciou-se que o tema "Conhecer a importância da homeopatia no SUS, os princípios da integralidade e da Política Nacional de Práticas Integrativas e Complementares - PNPIC", classificado de alto consenso, não obteve nota de relevância mais significativa por parte dos especialistas. Considerando que metade dos especialistas respondentes possui experiência docente superior a 20 anos, e que eles foram formados antes da implementação das DCN, os autores questionam se esses fatos poderiam ter influenciado na baixa relevância dada ao tema.

Os temas apontados pelos docentes especialistas, nesse trabalho, estão contidos em conteúdos programáticos de disciplinas de homeopatia na graduação, as quais na sua maioria são de caráter optativo, porém descritos somente de forma informativa ${ }^{10,27,31}$.

São praticamente inexistentes trabalhos a respeito dos conteúdos programáticos ou das competências conhecimentos e habilidades que devem ser ensinadas nas disciplinas de homeopatia, eosque existem sãovoltados para a pós-graduação. As diretrizes internacionais para a educação homeopática publicadas pelo Conselho Internacional de Homeopatia e pelo Conselho Central Europeu de Homeopatas em $2011^{32} \mathrm{e}$ os programas de residência médica em homeopatia do país elaborados pela $\mathrm{AMHB}^{33}$, ambos da especialização, abrangem 
temas e competências que vão além dos evidenciados por esse estudo. Viksveen et al. ${ }^{28}$ destacam a importância, segundo os educadores de homeopatia em relação a competências, do ensino centrado no estudante e que as habilidades estejam principalmente associadas à capacidade de comunicação interpessoal, empatia, observação individualizada, atitude centrada no paciente, além daquelas correlacionadas à teoria, pesquisa e filosofia homeopáticas e à capacidade prática da homeopatia ${ }^{28,32}$. Trata-se de características afins às desejadas para egresso médico descritas nas DCN.

A importância de construir uma matriz de competências sobre o que deve ser ensinado em homeopatia na graduação vem ao encontro do interesse dos médicos e estudantes de Medicina em aprender modalidades do que a Organização Mundial da Saúde denomina de Complementary and Alternative Medicines (CAM). No Brasil, as CAM são intituladas como Práticas Integrativas e Complementares (PIC), nas quais a homeopatia encontra-se incluída ${ }^{30,34}$. Segundo Teixeira ${ }^{7}$, alguns trabalhos nacionais e internacionais demonstram o grau de interesse dos atores da educação médica, tanto por parte dos discentes quanto dos docentes, em relação ao ensino das CAM e ao aprendizado dos seus fundamentos básicos, evidências científicas e abordagens clínico-terapêuticas. Países como Brasil, Estados Unidos, México, Reino Unido, Alemanha, Hungria, China, Irã, Austrália, entre outros, reiteram não somente a falta de conhecimentos dos discentes de Medicina e médicos residentes em relação às terapêuticas não convencionais durante a formação médica, como também o grande interesse em seu aprendizado ${ }^{7}$. Esses países, além de reiterarem a importância da homeopatia, insistem na inclusão do estudo dessa terapia no currículo médico convencional ${ }^{7,13}$.

No Brasil, algumas conquistas em âmbitos acadêmicos foram obtidas de forma pontual. A realidade é que ainda são poucas as ofertas do seu ensino tanto na graduação quanto na pós-graduação. Algumas faculdades públicas do país contemplam o ensino da homeopatia na graduação médica, porém ainda de forma heterogênea ${ }^{10,14,15,27}$.

Portanto, a proposta de se obter um consenso mínimo, objetivo e imprescindível do que deve ser ensinado ao graduando de Medicina vem no sentido de ajudar na orientação aos docentes e às IES na implantação e/ou no aperfeiçoamento do ensino da homeopatia na graduação médica.

Tanto as competências relacionadas aos conhecimentos quanto as habilidades que não obtiveram consenso alto conforme o índice de De Loe nem notas de relevância tão altas também devem ser objeto de reflexão não somente para os docentes que ministram uma disciplina de homeopatia, como para aqueles que desejam implementá-la de acordo com as características locais. Considera-se que a composição de uma matriz de competências a partir das avaliações desse grupo de especialistas, distribuídos por oito estados da Federação, pode ser muito útil nessa direção.

Contudo, para tal, cabe uma reflexão sobre a carga horária ideal para a aplicação da teoria e da prática, e também sobre o momento/período mais adequado para o conteúdo serministrado aos discentes. Apesar de o presente trabalho não ter previsto identificar as competências/atitudes, ficou clara a necessidade de desenvolvê-las, principalmente diante do relevante tema clínica homeopática, relacionado diretamente à prática, o que justifica a continuidade deste trabalho em estudos posteriores.

\section{CONCLUSÕES}

A elaboração de uma matriz de competências que sirva como uma recomendação do conteúdo epistemológico homeopático que deve ser ensinado aos discentes da graduação médica e que venha ao encontro do que é preconizado nas DCN para o curso de Medicina, além de contribuir na formação de um egresso com visão mais humanística e integral, propicia a oferta de conteúdos considerados essenciais pelos docentes especialistas, eliminando aquilo que é prescindível. Tal fato é relevante porque se considera que as cargas dos componentes curriculares do curso médico já são demasiadamente elevadas.

O ensino da homeopatia, que é uma especialidade médica no Brasil, pode contribuir para a mudança paradigmática da medicina no sentido de valorizar o doente e promover a saúde, permitindo uma atuação médica mais humanizada e centrada no doente, o que justificaria a adoção de seu ensino de forma obrigatória nas faculdades de Medicina.

Portanto, a construção dessa matriz composta de competências (conhecimentos e habilidades) torna-se um instrumento de reflexão na elaboração de uma futura ementa que possa servir como guia para aqueles docentes desejosos de implantar uma disciplina de homeopatia na graduação médica ou temas relacionados a ela dentro de uma disciplina afim em suas respectivas faculdades de Medicina.

\section{CONTRIBUIÇÃO DOS AUTORES}

Francisco José de Freitas participou da idealização e elaboração do projeto, da coleta de dados e análise dos dados, da análise dos resultados, da redação e revisão do texto. Maria Tereza Serrano Barbosa participou da elaboração do projeto, da orientação do trabalho, da análise dos dados, da análise dos resultados e da revisão do texto. Rodrigo da Fontoura de Albuquerque Mello participou da redação e revisão do texto.

\section{CONFLITO DE INTERESSES}

Informamos que não há qualquer conflito de interesses no presente trabalho. 


\section{FINANCIAMENTO}

Declaramos que não houve financiamento para a realização desta pesquisa.

\section{REFERÊNCIAS}

1. Brasil. Resolução CNE/CES n³/2014. Institui Diretrizes Curriculares Nacionais do Curso de Graduação em Medicina. Diário Oficial da União, Brasília; 23 jun 2014; Seção 1, p.8-11 [acesso em 12 set 2019]. Disponívelem:http://portal.mec. gov.br/index.php?option=com_docman\&view=download\&alias=15874rces003-14\&category_slug=junho-2014-pdf\&ltemid=30192.

2. Ribeiro LCC, Ribeiro $M$, Soares VAR. Avaliação acadêmica acerca das Diretrizes Curriculares Nacionais em Saúde. Tempus (Brasília). 2015;9(1):167-87.

3. Ben AJ, Lopes JMC, Daudt VG, Pinto MEB, Oliveira MMC. Rumo à educação baseada em competências: construindo a matriz do internato em Medicina de Família e Comunidade. Rev Bras Med Fam Comunidade. 2017;12(39):1-16.

4. Marinho-Araujo CM, Rabello ML. Avaliação educacional: a abordagem por competências. Rev Avaliação Educ Sup. 2015;20(2):443-66.

5. Gontijo ED, Alvim C, Megale L, Melo JRC, Lima MECC. Matriz de competências essenciais para a formação e avaliação de desempenho de estudantes de Medicina. Rev Bras Educ Med. 2013;37(4):526-39.

6. Ribeiro MMF, Amaral CSF. Medicina centrada no paciente e ensino médico: a importância do cuidado com a pessoa e o poder médico. Rev Bras Educ Med. 2008;32(1):90-7.

7. Teixeira MZ. Panorama mundial da educação médica em terapêuticas não convencionais (homeopatia e acupuntura). Rev Homeopatia (São Paulo). 2017;80(1-2):18-39.

8. Lehmann B, Kremer B, Werwick K, Hermann M. Homeopathy as elective in undergraduate medical education - an opportunity for teaching professional core skills. GMS Z Med Ausbild. 2014;31(1):1-15.

9. Salles SAC. Desenvolvimento de competências para uma abordagem integral do doente: a abordagem homeopática como referência na educação de estudantes de medicina. Rev Homeopatia (São Paulo). 2012;75(3-4):13-8

10. Salles SAC. A presença da homeopatia nas faculdades de Medicina brasileiras: resultados de uma investigação exploratória. Rev Bras Educ Med. 2008;32(3):283-90.

11. Brasil. Ministério da Saúde. Secretaria de Atenção Básica. Política Nacional de Práticas Integrativas e Complementares no SUS. 2 edição em 2015 [acesso em 12 set 2019 ]. Disponível em: http://bvsms.saude. gov.br/bvs/publicacoes/politica_nacional_praticas_integrativas_ complementares_2ed.pdf.

12. Teixeira MZ. Possíveis contribuições do modelo homeopático à humanização da formação médica. Rev Bras Educ Med. 2009;33(3):465-74.

13. Fernandes DAS, Freitas FJ, Guimaraes RGM, Haege DP. Avaliação da relevância do ensino da homeopatia no curso de graduação de medicina da UNIRIO. Rev Homeopatia (São Paulo). 2011; 74(3):14 [acesso em 21 Sept 2019]. Disponível em: http://revista.aph.org.br/index.php/aph/article/ view/81/202.

14. Freitas FJ, Fernandes DAS, Mello RFA, Sollero C, Carvalho CSCC. Evolução e relevância da Homeopatia na UNIRIO. Rev Homeopatia (São Paulo). 2011;74(3):23 [acesso em 21 Sept 2019]. Disponível em: http://revista.aph. org.br/index.php/aph/article/view/89/158.

15. Deus RL. Trabalhadores da atenção primária à saúde e as práticas integrativas e complementares - do uso à indicação [dissertação]. Juiz de Fora: Universidade Federal de Juiz de Fora; 2016.
16. Humphrey-Murto S, Varpio L, Wood TJ, Gonsalves C, Ufholz MA, Mascioli $\mathrm{K}$, et al. The use of the Delphi and other consensus group methods in medical education research: a review. Acad Med. 2017;92(10):1491-8.

17. Valente AAMO, Caldato MCF. Matriz de competências para programa de residência médica em endocrinologia e metabologia. Rev Bras Educ Med 2019;43(supl.1):207-18.

18. Revoredo LS, Maia RS, Torres GV, Maia EMC. O uso da técnica Delphi em saúde: uma revisão integrativa de estudos brasileiros. Arq Ciênc Saúde. 2015;22(2):16-21.

19. Rozados HBF. O uso da técnica Delphi como alternativa metodológica para a área da Ciência da Informação. Em Questão. 2015;21(3):64-86.

20. Shimizu HE, Fragelli TBO. Competências profissionais essenciais para o trabalho no núcleo de apoio à saúde da família. Rev Bras Educ Med. 2016;40(2):216-25.

21. Pita CG. Matriz de competência para residência médica em cardiologia da Universidade Federal do Rio Grande do Norte [dissertation]. Natal: Universidade Federal do Rio Grande do Norte; 2018.

22. Marques JBV, Freitas D. Método DELPHI: caracterização e potencialidades na pesquisa em educação. Pro-Posições. 2018;29(2):389-415.

23. De Loe RC. Exploring complex policy questions using the policy Delphi. Appl Geogr. 1995;15(1): 53-68.

24. Powell C. The Delphi technique: myths ande realities. J Adv Nurs 2003:41(4):376-82 [acesso em 15 set 2019]. Disponível em: https://pdfs. semanticscholar.org/0707/aecd593c8eef182dd417bcb37e207edf4c3d.pdf.

25. Osborne J, Collins S, Ratcliffe M, Millar R, Duschl R. What "Ideas-aboutScience" should be taught in school science? A Delphi study of the expert community. J Res Sci Teach. 2003;40(7):692-720.

26. Scheffer M, Cassenote A, Guilloux AGA, Biancarelli A, Miotto BA, Mainard GM. Demografia Médica no Brasil 2018. São Paulo: Faculdade de Medicina da USP, Conselho Federal de Medicina, Conselho Regional de Medicina do Estado de São Paulo; 2018 [acesso em 30 Aug 2020]. Disponível em: http:// jornal.usp.br/wp-content/uploads/DemografiaMedica2018.pdf.

27. Oliveira IFO, Peluso BHB, Freitas FAC, Nascimento MC. Homeopatia na graduação médica: trajetória da Universidade Federal Fluminense. Rev Bras Educ Med. 2018;42(1):92-102.

28. Viksveen $P$, Steinsbekk A, Rise MB. What is a competent homeopath and what do they need in their education? A qualitative study of educators' views. Educ Health. 2012;25(3):172-9.

29. Santos WS. Organização curricular baseada em competência na educação médica. Rev Bras Educ Med. 2011;35(1):86-92.

30. Pustiglione M, Goldenstein E, Checinski M. Homeopatia: um breve panorama desta especialidade médica. Rev Homeopatia (São Paulo). 2017;80(1-2):1-17.

31. Universidade Federal do Estado do Rio de Janeiro. Projeto Pedagógico do curso de Medicina. Rio de Janeiro: Unirio; 2014 [acesso em 10 abr 2019] Disponível em: http://www.unirio.br/emc/ProjetoPedagogicoMedicina. corrigido.pdf

32. European Central Council of Homeopaths. The International Guidelines for Homeopathy Education, An ECCH and ICH document. May 2011 [acesso em 10 abr 2019]. Disponível em: http://www.homeopathy-ecch.org/wpcontent/uploads/2017/02/ECCH-PROFESSIONAL-PORTFOLIO-2017.pdf.

33. Brasil. Ministério da Educação. Comissão Nacional de Residência Médica. Programa de Residência Médica em Homeopatia [access em 10 abr 2019]. Disponível em: http://portal.mec.gov.br/index. php?option=com_docman\&view=download\&alias=6516-homeopatiasesu-rm\&ltemid=30192

34. World Health Organization. Traditional Medecine Strategy: 2014-2023 [acesso em 15 out 2019]. Disponível em: http://www.searo.who.int/entity/ health_situation_trends/who_trm_strategy_2014-2023.pdf?ua=1. 